\title{
Recent Cosmological Anisotropy Explained by Dark Energy as Universes of Negative Gravitational Mass
}

\author{
Stéphane Le Corre \\ Ecole Polytechnique Fédérale de Lausanne, Route Cantonale, Lausanne, Switzerland \\ Email: le.corre.stephane@hotmail.fr
}

How to cite this paper: Le Corre, S. (2021) Recent Cosmological Anisotropy Explained by Dark Energy as Universes of Negative Gravitational Mass. Open Access Library Journal, 8: e7587.

https://doi.org/10.4236/oalib.1107587

Received: May 26, 2021

Accepted: June 21, 2021

Published: June 24, 2021

Copyright $\odot 2021$ by author(s) and Open Access Library Inc.

This work is licensed under the Creative Commons Attribution International License (CC BY 4.0).

http://creativecommons.org/licenses/by/4.0/

\section{(c) (i) Open Access}

\begin{abstract}
A recent publication finds strong evidence for a violation of the Cosmological principle of isotropy across the CMB sky. This analysis revealed three distinct patches in the maps with circularly-averaged sizes between 40 to 70 degrees in radius. These three areas distinguish large-scale deviations from the all-sky mean value of several distinguished cosmological parameters measured separately. In this article, we analyze these results with a previous solution proposed to explained dark energy consistent with general relativity. This solution allows retrieving all the qualitative and quantitative observations ( 3 areas with the same kind of deviations from all-sky mean values, with circular boundaries, with right size and position, consistent with the sign of deviation for the Hubble parameter $H_{0}$ for each area).
\end{abstract}

\section{Subject Areas}

Classical Physics, Mathematical Analysis

\section{Keywords}

Cosmic Background Radiation, Dark Energy, Large-Scale Structure of Universe

\section{Introduction}

As mentioned in [1], the analysis of CMB data, most notably from the WMAP [2] and Planck [3] experiments, has not yet provided conclusive evidence for the hypothesis of Cosmological Isotropy ([4]-[9]; see also [10] and references therein). However, a more recent analysis based on Planck data finds no evidence for such power asymmetry when all scales are considered [11] but by using a statis- 
tical prior. Alternatively, using no prior in their approach, in [1], their analysis shows compelling statistical evidence for large-scale anisotropies (Gaussian isotropic hypothesis has a probability of $\sim 10^{-9}$ ), sourced by large-scale directional variations in all the basic $\Lambda \mathrm{CDM}$ parameters.

Concretely, their analysis revealed three areas in which the values of several cosmological parameters are either superior to the all-sky mean values or inferior. For each cosmological parameter analyzed, these three areas are similar. They denoted these areas, $\mathrm{H} 1, \mathrm{H} 2$, and $\mathrm{H} 3$ as in Figure 1.

Qualitatively, this analysis observed that:

1) There are three main areas of variations of the cosmological parameters in the $\mathrm{CMB}$ data ( $\mathrm{H} 1, \mathrm{H} 2$ and $\mathrm{H} 3)$.

2) The boundary of the areas (when the parameter variation vanishes) is circular.

3) $\mathrm{H} 1$ and $\mathrm{H} 3$ are the same kind of variations (superior or inferior to the mean value) for each cosmological parameter.

4) Inversely, $\mathrm{H} 2$ are systematically an opposite behavior from $\mathrm{H} 1$ and $\mathrm{H} 3$ for each cosmological parameter.

5) The Hubble parameter $H_{0}$ are lower than all-sky mean in $\mathrm{H} 2$ and greater in $\mathrm{H} 1$ and $\mathrm{H} 3$.

6) The dark energy parameter $\Delta \Omega_{\Lambda}$ are lower than all-sky mean in $\mathrm{H} 2$ and greater in $\mathrm{H} 1$ and $\mathrm{H} 3$.

7) The circularly-averaged sizes are rough of the same order of magnitude around $55^{\circ}$ (between 40 to 70 degrees in radius).

8 ) The areas partially overlap between them.

The four points $(3,4,5$ and 6$)$ certainly mean that the source of deviations for $\mathrm{H} 1$ and $\mathrm{H} 3$ are similar properties but not for $\mathrm{H} 2$. These observations are in fact very structuring observations that constraints enormously the idealization as we discuss it in the section "Discussion".

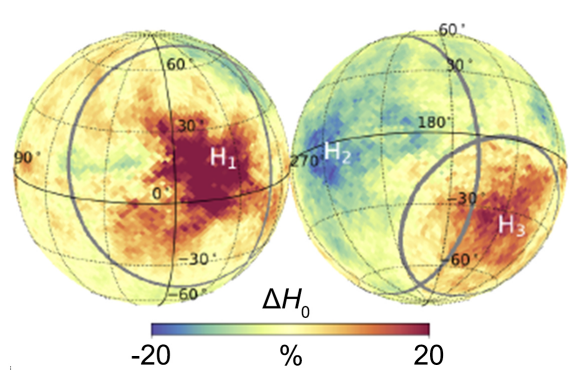

(a)

(b)

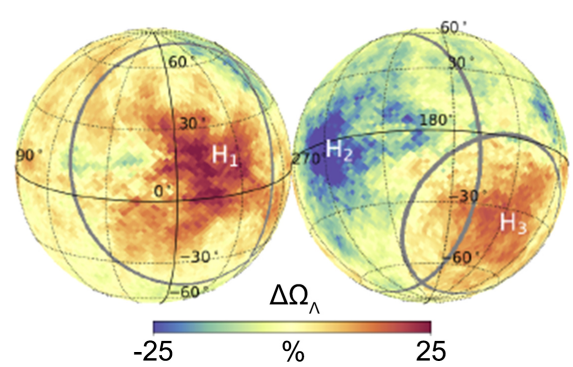

(c)

(d)

Figure 1. From [1]: Data shown in orthographic projection with the left hemisphere, (a) and (c), centered at the Galactic center and the right hemisphere, (b) and (d), around the anti-Galactic center. On (a) and (b), the three horizons (denoted by H1, H2 and H3) identified across the Hubble parameter variation map. The circular boundary of each horizon is set when the parameter variation vanishes, i.e., when it is equal to the all-sky mean (in light yellow for the color scale used). Note that the horizons partially overlap between them. Very similar horizons are obtained for the other $\Lambda$ CDM parameters (for example on (c) and (d) for the Dark-Energy density parameter). 
Quantitatively, they obtain:

9) the center position of $\mathrm{H} 1$ in longitude and latitude around $\left(345^{\circ}, 15^{\circ}\right)$.

10) the circularly-averaged sizes of $\mathrm{H} 1$ around $60^{\circ}$ in radius.

11) the center position of $\mathrm{H} 2$ in longitude and latitude around $\left(240^{\circ} ;-5^{\circ}\right)$.

12) the circularly-averaged sizes of $\mathrm{H} 2$ around $70^{\circ}$ in radius.

13) the center position of $\mathrm{H} 3$ in longitude and latitude around $\left(150^{\circ} ;-50^{\circ}\right)$.

14) the circularly-averaged sizes of $\mathrm{H} 3$ around $40^{\circ}$ in radius.

Hereafter, we are going to study how a solution that has been proposed in can explain these fourteen observations. This solution has been proposed to explain dark energy in the frame of General Relativity. But let's first remind the main ideas of this solution.

\section{Dark Energy Explained by General Relativity with Negative Gravitational Mass}

A solution is proposed to explain dark energy in agreement with general relativity. This solution considers the assumption of the existence of negative gravitational mass (with always positive inertial mass). The negative gravitational masses would be attractive to each other (as for the positive gravitational masses) but a negative mass and a positive mass would be repulsive. One can find articles about negative mass but they all consider that negative mass will gravitationally repel all surrounding masses [12] [13] [14], leading to the very problematic case referred as runaway motion. As explained in, because only gravitational mass can be negative all the physical issues are resolved. It can be easily understood when general relativity is linearized [15]. Linearized general relativity is then similar to electromagnetism but with "symmetrical" effects, i.e. attractive between particles with gravitational masses of the same sign, repulsive between particles with gravitational masses of opposite sign. In this way, one can understand that negative mass is physically consistent (just like Maxwell's equations are). But unlike electromagnetism tends to neutralize its aggregations at a small scale (at the scale of the molecule), gravitation aggregates homogenous masses at a large scale (at the scale of the universe).

In [15], it is also shown that necessarily, if negative gravitational masses exist, the antiparticles must have negative gravitational masses. Experiments are in progress (in particular at CERN with AEGIS, GBAR, ...) to test this hypothesis. Several consequences of this hypothesis are also predicted. First, the existence of many other universes of matter beyond our own Universe, second, the existence of universes of only antimatter (similar to our Universe of only matter), and third, a structure of universes alternately of matter and of antimatter. Simulations would be necessary to determine which kind of networks can appear from a cloud of positive and negative particles in which attractive and repulsive force acts following the equations of [15], coming from the linearized general relativity and similar to the Maxwell's equations. Naturally, a period of segregation of masses occurs, aggregating large and regular homogenous masses (explaining 
the absence of negative mass in our Universe). The cloud is then going to find a steady arrangement by alternating large areas of positive and negative gravitational masses. At first approximation, a possible solution could look like 2 entangled cubic networks (Figure 2). We will see at the end of this paper that the results of [1] allow thinking that the cubic network is maybe too basic but nevertheless it allows obtaining the observations, certainly meaning that the right networks are not so far to this arrangement.

In this basic approximation, it is natural to expect that these large areas of positive and negative gravitational masses (the universes) are of the same order of size, with similar cosmological parameters and expanding at the same rate. Because of the attractive and repulsive gravitation, at this approximation one can consider that no matter is between these universes. The influence of neighboring universes of opposite gravitational masses would produce the opposite pressure which could explain the cosmic acceleration.

\section{Negative Gravitational Mass and Areas of Distinct Values of Comological Parmeters in Our Universe}

The results observed in [1] fit surprisingly well with this structure, despite the extreme simplicity of the approximation of the article. First, on the left of Figure 3 , let's consider in this structure, the closest neighbors of our Universe, that is 8 universes of antimatter and 6 universes of matter (slightly less close than antimatter). And then, on the right of Figure 3, let's consider that our observable universe which is a restricted area of our own whole Universe is located near a boundary (near an edge of the cubic network).

With this very basic idealization and with the very constraining assumptions that all the universes are identical (same size, same density, same cosmological parameters...excepted the sign of the gravitational mass) and centered on the nodes of the 2 cubic networks, one retrieves the main characteristics of the observation of the anisotropies. This assumption of identical universes is constraining because it makes our idealization not very modifiable (and more mathematical than physical). But the advantage is that it is very simple to manipulate. We will see further that by releasing these constraints (for example only moving the universes from their network nodes that is a more reasonable physical assumption), this idealization can match exactly with the observations. But
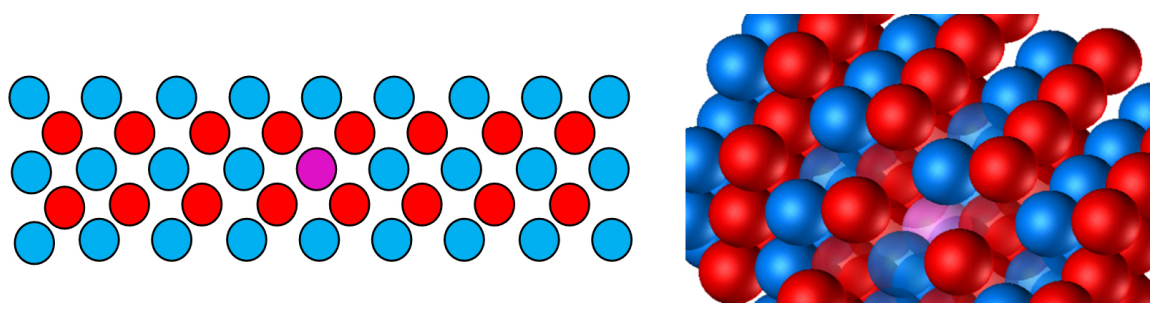

Figure 2. Network, in 2D on the left and in 3D on the right, of positive (blue spheres) and negative (red spheres) gravitational masses. Each zone (red or blue spheres) represent a universe similar to our own universe (pink sphere). 

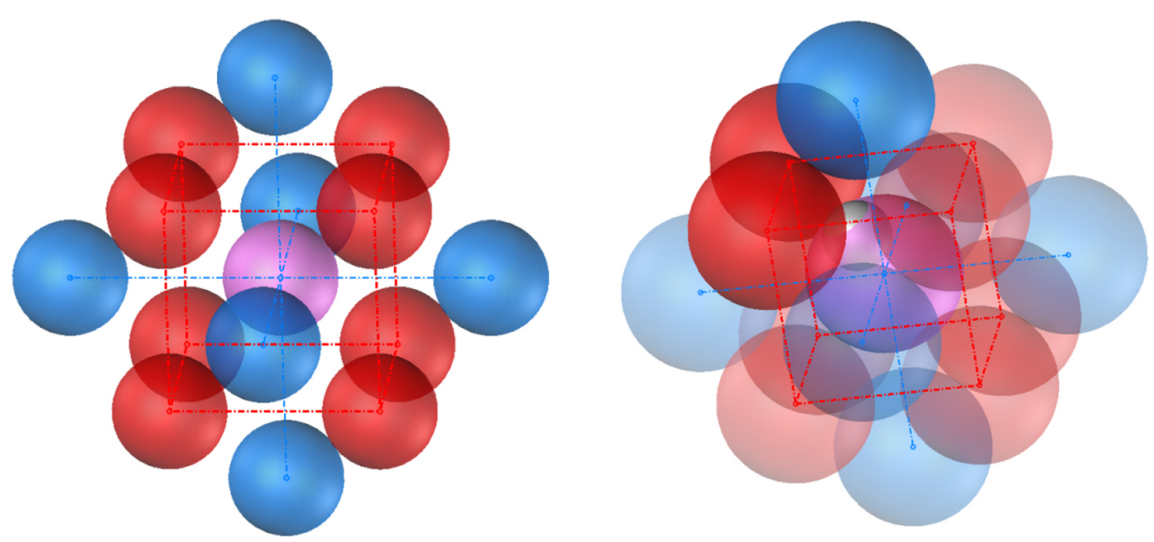

Figure 3. On the left, our Universe (pink sphere) surrounded by 8 closest universes of antimatter (red spheres) and 6 less close universes of matter, due to imbrication of 2 cubic networks. On the right, our observable universe measured in the CMB data (grey sphere) included in our whole Universe and located near an edge of the cubic network. The grey sphere is shown slightly outside the pink one only to make it more visible.

by this way, one can avoid any calculation and find geometrical solutions. Indeed, to show the areas of influence of our neighboring universes, we can increase the spheres (which until now represent the universes in which there is some matter) to become spheres of influence of our universes and show the intersection of these spheres. The surfaces of these spheres of influence represent then the surface of "iso-gravitation" just like the contour lines of a 2D geographical map. It doesn't represent an overlap of the matter of the universes that is not expected in this solution. In Figure 4, we can see on left, the areas of influence of neighboring universes on our own whole Universe and on the right the influence on our observable universe.

The first observation is that it remains only three areas of notable influence for our observable universe in this location inside our own whole Universe. It should be understood that it doesn't remain 3 areas not because we subjectively decided to take in account only these three universes but because for our observable universe the other universes are far enough to have their sphere of influence not in intersection with our observable sphere, meaning that their gravitational influence is less important than in these three areas (and given their distance from our sphere, most certainly negligible in a first approximation). Furthermore, because of this "iso-gravitational" sphere, the natural areas of influence have circular boundaries. These facts explain the first two points observed in [1]:

1) There are three main areas of variations of the cosmological parameters in the $\mathrm{CMB}$ data ( $\mathrm{H} 1, \mathrm{H} 2$ and $\mathrm{H} 3)$.

2) The boundary of the areas (when the parameter variation vanishes) is circular.

Furthermore, at this location two of these spheres of influences are due to antimatter universes (denoted $\mathrm{H} 1$ and $\mathrm{H} 3$ in Figure 4 on the right) and the third sphere due to matter universe (denoted $\mathrm{H} 2$ in Figure 4 on the right). It implies 

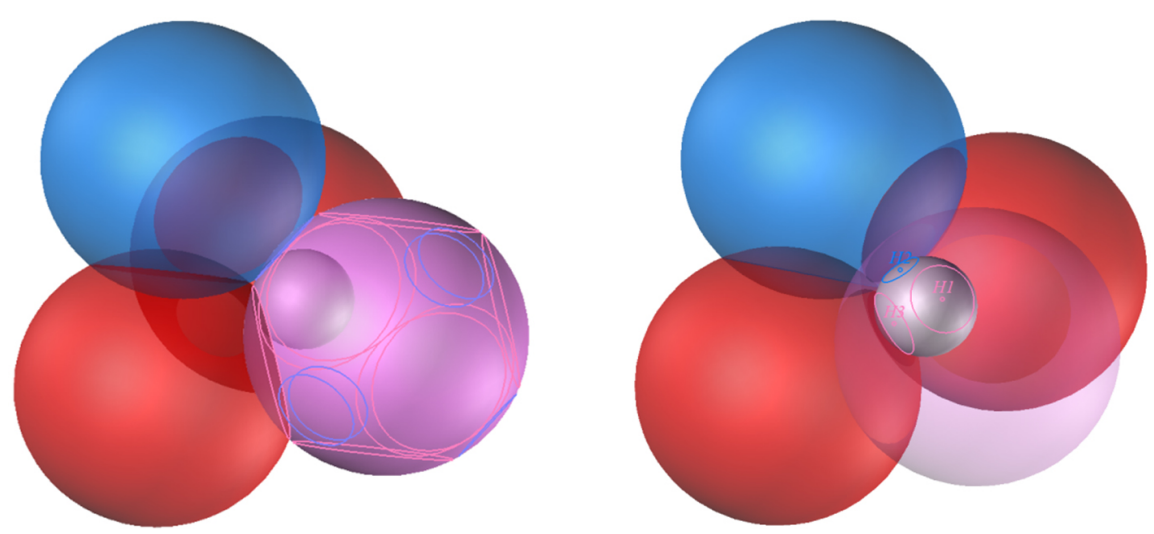

Figure 4. On the left, areas of influence of closest universes (8 of antimatter and 6 of matter) on our Universe (pink sphere) and on the right on our observable universe (grey sphere).

that the cosmological parameters in $\mathrm{H} 1$ and $\mathrm{H} 3$ will be deviated in the same sense (relatively to the all-sky mean) and in $\mathrm{H} 2$ in the opposite sense because antimatter (red spheres) is repulsive for our Universe of matter. And because of this gravitational repulsion, the Hubble parameter $H_{0}$ must be greater in $\mathrm{H} 1$ and H3. These universes of antimatter are in fact the explanation of the acceleration of the expansion of our universe. And because anti-universes are the source of dark energy in this solution, it also explains the increase of dark energy parameter $\Delta \Omega_{\Lambda}$ measured in Figure 1 for $\mathrm{H} 1$ and $\mathrm{H} 3$. This fact explains four other points observed in [1]:

3) $\mathrm{H} 1$ and $\mathrm{H} 3$ are the same kinds of variations (superior or inferior to the mean value) for each cosmological parameter.

4) Inversely, $\mathrm{H} 2$ are systematically an opposite behavior from $\mathrm{H} 1$ and $\mathrm{H} 3$ for each cosmological parameter.

5) The Hubble parameter $H_{0}$ are lower than all-sky mean in $\mathrm{H} 2$ and greater in $\mathrm{H} 1$ and $\mathrm{H} 3$.

6) The dark energy parameter $\Delta \Omega_{\Lambda}$ are lower than all-sky mean in $\mathrm{H} 2$ and greater in $\mathrm{H} 1$ and $\mathrm{H} 3$.

As written before, these 4 points are certainly the more constraining observations. And the fact that this solution naturally implies these observations is a very strong result of this idealization.

In this solution, universes are physically identical, it explains why the size of the areas of influence are of the same order of magnitude. This fact explains another qualitative point observed in [1]:

7) The circularly-averaged sizes are roughly of the same order of magnitude.

Let's now see if with these constraining assumptions and very basic idealization, one can retrieve roughly the position and the sizes of these areas (with more accuracy than an order of magnitude). In Figure 5, one has only modified the location of our observable universe to slightly enhanced the position of the areas $\mathrm{H} 1, \mathrm{H} 2$ and $\mathrm{H} 3$. By this way, we obtain the last qualitative point observed in [1]: 

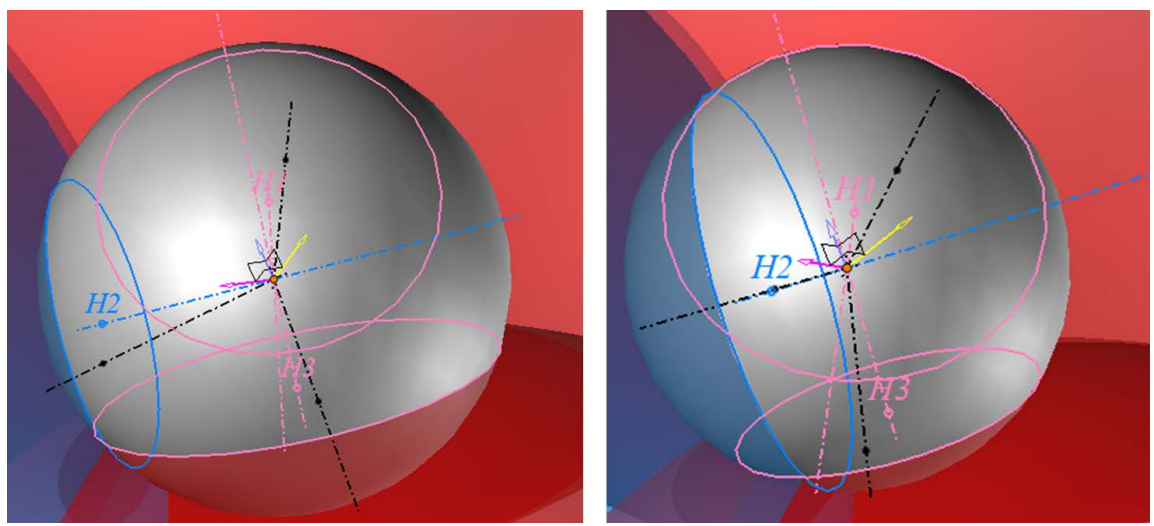

Figure 5. Depending on the position of our observable universe, on left, the centers' location's fit is better than the radius of the areas, on the right the radius of the areas' fit is better than the centers' location. For a physical idealization more realistic, some constraints must be released and fortunately it then allows a better accuracy.

8) The areas partially overlap between them.

One can also remark that this overlap could be obtained with a larger sphere of influence (this parameter defining in fact the quantity of matter of the universes).

One can observe, that despite our constraining assumptions, we obtain the centers of these areas measured in [1], represented by the black points, in the expected areas roughly close to the geometrical center (denoted $\mathrm{H} 1, \mathrm{H} 2$ and $\mathrm{H} 3$ on Figure 5).

These locations are not so bad considering the many constraints of our idealizations that allow resolving easily our solution but with a loss of accuracy. By releasing the constraints, one can enhance this idealization. If the size of neighboring universe is not strictly identical for all, we can adjust the circularlyaveraged sizes of the 3 areas independently each other. And if the position of the universes is not strictly a cubic network, the center position of the 3 areas can also be adjusted. For example, in Figure 6, one obtains the right circularlyaveraged sizes and the right position of centers for the 3 areas $\mathrm{H} 1, \mathrm{H} 2$ and $\mathrm{H} 3$ by only moving the 2 anti-universes (pink spheres) from their node of cubic network.

By this way, we obtain the quantitative points observed in [1]:

9) the center position of $\mathrm{H} 1$ in longitude and latitude around $\left(345^{\circ}, 15^{\circ}\right)$.

10) the circularly-averaged sizes of $\mathrm{H} 1$ around $60^{\circ}$ in radius.

11) the center position of $\mathrm{H} 2$ in longitude and latitude around $\left(240^{\circ} ;-5^{\circ}\right)$.

12) the circularly-averaged sizes of $\mathrm{H} 2$ around $70^{\circ}$ in radius.

13) the center position of $\mathrm{H} 3$ in longitude and latitude around $\left(150^{\circ} ;-50^{\circ}\right)$.

14) the circularly-averaged sizes of $\mathrm{H} 3$ around $40^{\circ}$ in radius.

\section{Discussion}

The solution showed in Figure 6 is obtained by moving the 2 anti-universes (pink spheres) from their node to get a greater distance than initially expected. 


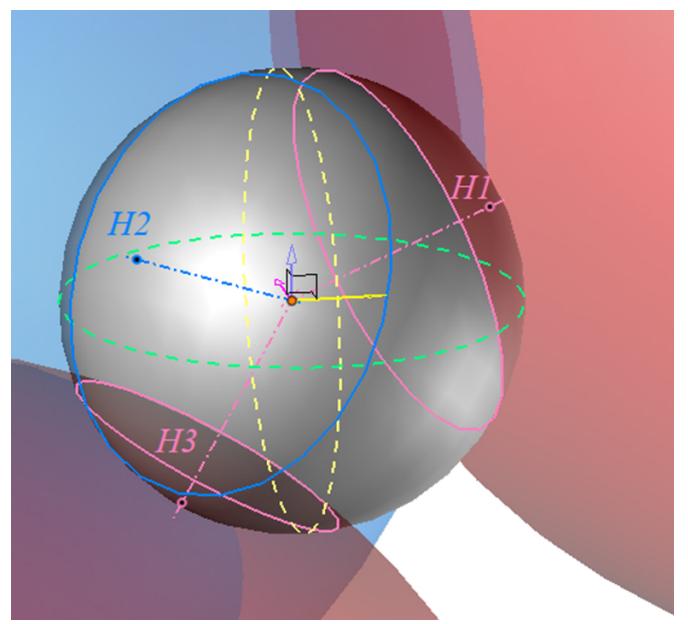

Figure 6. Example of a solution allowing obtaining the areas $\mathrm{H} 1, \mathrm{H} 2$ and $\mathrm{H} 3$ with the right circularly-averaged sizes and the right position of centers observed in [1]. In this solution, the universes are moved from nodes of the cubic network.

This fact is consistent with the expected physical situation. Indeed, as explained in that this explanation of dark energy leads most likely to a damping phenomenon with an oscillation of the universes around their theoretical steady positions. By this way, it means then that first, the distance between antimatter's universes should be different than between matter's universes and secondly that the "accelerator" effect should change with time. It would be in agreement with the discrepancy that seems appearing on the Hubble's constant measured values [16] between measures at the $\mathrm{CMB}$ time and at more recent time (closer to us). In this explanation, there would be two main contradictories effects with different specific own time (natural frequency), one which tends to increase the acceleration (by increasing the mass and size of the universes during the time of the masses segregation until reaching a typical size and mass of universe) and another which tends to decrease the acceleration (by increasing the distance between universes of masse of opposite sign) (Figure 7). It would be then very interesting to see the evolution of these 3 areas $\mathrm{H} 1, \mathrm{H} 2$ and $\mathrm{H} 3$ along the time until recent time. It would inform on the dynamic of our neighboring universes.

The solution proposed can obtain the results observed in [1] but in [1], another solution [17] [18] [19] is mentioned that can also explain these observations.

In these two solutions, our own Universe is surrounded by others universes. But there are two main differences. These neighboring universes are alternatively of negative and positive gravitational masses, in [17] [18] [19] they are only of positive gravitational mass. And the networks of these universes are not the same in the two solutions. For the first point, some experiments in CERN (AEGIS, GBAR, ...) will soon allow validating or not the assumption of negative gravitational masses. About the network of the universes, one can make a criticism of the solution. Even if the solution can explain the observations, the location of our observable universe is very eccentric and perhaps too eccentric. 


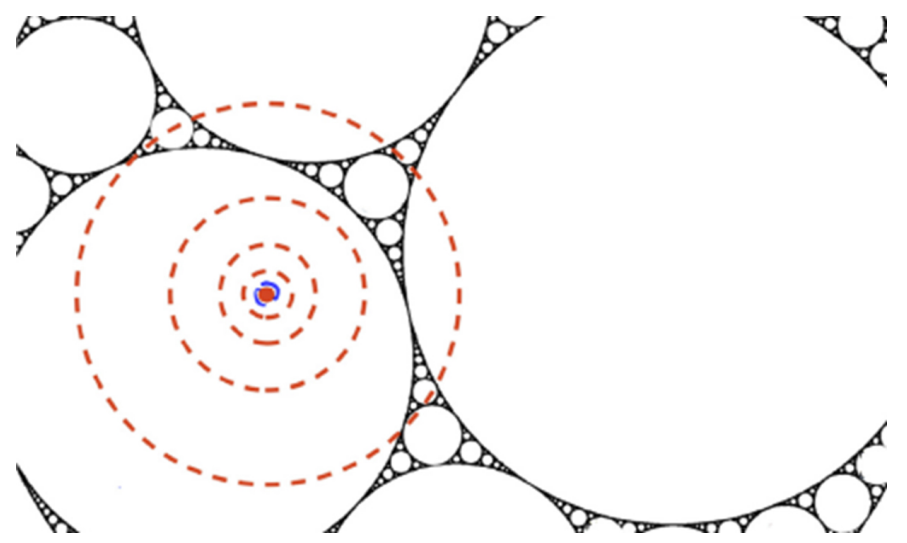

Figure 7. Illustration of the causal structure of the Universe after inflation. Each circle (with continuous lines) represents an independent Universe: a causally disconnected horizon with different cosmological parameters. Our galaxy is depicted at the centre of the dashed concentric circles that represent the growth evolution of our particle horizon after inflation ends.

But this solution could be enhanced with a double network not so basic than cubic networks. Pentagonal or even hexagonal networks allow localizing our own observable universe in a less eccentric location by enlarging the volume of the mesh (with the same edge length of the network tested in the current article to maintain the results).

\section{Conclusions}

Dark energy is explained in agreement with general relativity as the effects of negative gravitational mass (repulsive with positive gravitational mass and attractive with negative gravitational mass). Experiments are in progress (in particular at CERN with AEGIS, GBAR, ...) to test this assumption. This explanation implies the creation of pairs of opposite gravitational masses (particleantiparticle) at the apparition of the gravitational interaction (at the first age of the Universe). This cloud of pairs generates necessary primordial inflation (because of the repulsive gravitational force between the opposite gravitational masses inside each pair). This inflation is followed by a segregation period during which large zones of homogeneous masses are structured. These large zones of gravitational mass of the same sign (one of which is our Universe) make attractive gravitational interaction dominate at a large scale (at the scale of our Universe). But at the scale beyond one universe, expansion with segregation period lead to a fragmentation of the initial cloud of pairs of opposite gravitational masses into large zones of homogeneous gravitational mass which structures an imbrication of a network of universes of positive gravitational masses and a network of universes of negative gravitational masses (universes of antiparticles). In a first approximation, a possible mathematical solution can be a double cubic network of alternatively universes of positive and negative gravitational masses. We show that all the observations described in [1], are explained in the context of this solution of dark energy: 
1) There are three main areas of variations of the cosmological parameters in the $\mathrm{CMB}$ data $(\mathrm{H} 1, \mathrm{H} 2$, and $\mathrm{H} 3)$.

2) The boundary of the areas (when the parameter variation vanishes) is circular.

3) $\mathrm{H} 1$ and $\mathrm{H} 3$ are the same kinds of variations (superior or inferior to the mean value) for each cosmological parameter.

4) Inversely, $\mathrm{H} 2$ are systematically an opposite behavior from $\mathrm{H} 1$ and $\mathrm{H} 3$ for each cosmological parameter. It certainly means that the source of deviations for $\mathrm{H} 1$ and $\mathrm{H} 3$ are similar properties but not for $\mathrm{H} 2$. It will be clearly the case in our solution.

5) The Hubble parameter $H_{0}$ are lower than all-sky mean in $\mathrm{H} 2$ and greater in $\mathrm{H} 1$ and $\mathrm{H} 3$.

6) The dark energy parameter $\Delta \Omega_{\Lambda}$ are lower than all-sky mean in H2 and greater in $\mathrm{H} 1$ and $\mathrm{H} 3$.

7) The circularly-averaged sizes are rough of the same order of magnitude around $55^{\circ}$ (between 40 to 70 degrees in radius).

8) The areas partially overlap between them.

9) The center position of $\mathrm{H} 1$ in longitude and latitude around $\left(345^{\circ}, 15^{\circ}\right)$.

10) The circularly-averaged sizes of $\mathrm{H} 1$ around $60^{\circ}$ in radius.

11) The center position of $\mathrm{H} 2$ in longitude and latitude around $\left(240^{\circ} ;-5^{\circ}\right)$.

12) The circularly-averaged sizes of $\mathrm{H} 2$ around $70^{\circ}$ in radius.

13) The center position of $\mathrm{H} 3$ in longitude and latitude around $\left(150^{\circ} ;-50^{\circ}\right)$.

14) The circularly-averaged sizes of $\mathrm{H} 3$ around $40^{\circ}$ in radius.

One of the strengths of this solution is not only the determination of the areas of variations of the cosmological parameters but also the explanation of the sign of the variations (lower or greater than all-sky mean) of the cosmological parameters in each area (the Hubble parameter $H_{0}$ and the dark energy parameter $\left.\Delta \Omega_{\Lambda}\right)$.

The capacity of the solution described to explain the results of [1] reveals the relevance of the assumption of dark energy as negative gravitational mass and consequently could be an indirect clue of the existence of negative gravitational mass. Let's remind that this assumption allows explaining cosmic inflation, the onset of the radiation epoch, and the reheating epoch [20].

To end let's make a last remark. With the results of [1], the authors finally indirectly "observe" the universes beyond our own observable universe. This prospect is quite formidable.

\section{Conflicts of Interest}

The author declares no conflicts of interest regarding the publication of this paper.

\section{References}

[1] Fosalba, P. and Gaztañaga, E. (2021) Explaining Cosmological Anisotropy: Evidence for Causal Horizons from CMB Data. Monthly Notices of the Royal Astronomical 
Society, 504, 5840-5862. https://doi.org/10.1093/mnras/stab1193

[2] Bennett, C.L., et al. (2013) Nine-Year Wilkinson Microwave Anisotropy Probe (WMAP) Observations: Final Maps and Results. The Astrophysical Journal Supplement, 208, 54 p. https://doi.org/10.1088/0067-0049/208/2/20

[3] Alves, J., Forveille, T., Pentericci, L. and Shore, S. (2020) Planck 2018 Results. I. Overview and the Cosmological Legacy of Planck. $A \& A, 641$, Article No. A1. https://www.aanda.org/articles/aa/full_html/2020/09/aa33880-18/aa33880-18.html

[4] Eriksen, H.K., Hansen, F.K., Banday, A.J., Górski, K.M. and Lilje, P.B. (2004) Asymmetries in the Cosmic Microwave Background Anisotropy Field. The Astrophysical Journal, 605, 14-20. https://doi.org/10.1086/382267

[5] Hajian, A., Souradeep, T. and Cornish, N. (2005) Statistical Isotropy of the Wilkinson Microwave Anisotropy Probe Data: A Bipolar Power Spectrum Analysis. The Astrophysical Journal, 618, L63-L66. https://doi.org/10.1086/427652

[6] Eriksen, H.K., Banday, A.J., Górski, K.M., Hansen, F.K. and Lilje, P.B. (2007) Hemispherical Power Asymmetry in the Third-Year Wilkinson Microwave Anisotropy Probe Sky Maps. The Astrophysical Journal, 660, L81-L84. https://doi.org/10.1086/518091

[7] Land, K. and Magueijo, J. (2007) The Axis of Evil Revisited. Monthly Notices of the Royal Astronomical Society, 378, 153-158. https://doi.org/10.1111/j.1365-2966.2007.11749.x

[8] Hansen, F.K., Banday, A.J., Gorski, K.M., Eriksen, H.K. and Lilje, P.B. (2009) Power Asymmetry in Cosmic Microwave Background Fluctuations from Full Sky to Sub-Degree Scales: Is the Universe Isotropic? The Astrophysical Journal, 704, 1448-1458. https://doi.org/10.1088/0004-637X/704/2/1448

[9] Samal, P.K., Saha, R., Jain, P. and Ralston, J.P. (2009) Signals of Statistical Anisotropy in WMAP Foreground-Cleaned Maps. Monthly Notices of the Royal Astronomical Society, 396, 511-522. https://doi.org/10.1111/j.1365-2966.2009.14728.x

[10] Planck Collaboration et al. (2020) Planck 2018 Results. VII. Isotropy and Statistics of the CMB. $A \& A, 641$, Article No. A7.

https://www.aanda.org/articles/aa/full_html/2020/09/aa35201-19/aa35201-19.html

[11] Quartin, M. and Notari, A. (2015) On the Significance of Power Asymmetries in Planck CMB Data at All Scales. Journal of Cosmology and Astroparticle Physics, 2015, Article No. 008. https://doi.org/10.1088/1475-7516/2015/01/008

[12] Socas-Navarro, H. (2019) Can a Negative-Mass Cosmology Explain Dark Matter and Dark Energy? $A \& A, 626$, Article No. A5.

https://doi.org/10.1051/0004-6361/201935317

https://ui.adsabs.harvard.edu/abs/2019A\%26A...626A...5S/abstract

[13] Farnes, J.S. (2018) A Unifying Theory of Dark Energy and Dark Matter: Negative Masses and Matter Creation Within a Modified $\Lambda$ CDM Framework. $A \& A, 620, \mathrm{Ar}$ ticle No. A92. https://doi.org/10.1051/0004-6361/201832898 https://ui.adsabs.harvard.edu/abs/2018A\%26A...620A..92F/abstract

[14] Bonnor, W.B. (1989) Negative Mass in General Relativity. General Relativity and Gravitation, 21, 1143-1157. https://doi.org/10.1007/BF00763458 https://ui.adsabs.harvard.edu/abs/1989GReGr..21.1143B/abstract

[15] Le Corre, S. (2018) About the Negative Gravitational Mass. Open Access Library Journal, 51, e4312. https://doi.org/10.4236/oalib.1104312

[16] Riess, A., et al. (2021) Cosmic Distances Calibrated to 1\% Precision with Gaia EDR3 
Parallaxes and Hubble Space Telescope Photometry of 75 Milky Way Cepheids Confirm Tension with $\Lambda$ CDM. The Astrophysical Journal Letters, 908, $11 \mathrm{p}$. https://doi.org/10.3847/2041-8213/abdbaf

[17] Gaztañaga, E. (2020) The Size of Our Causal Universe. Monthly Notices of the Royal Astronomical Society, 494, 2766-2772.

https://doi.org/10.1093/mnras/staa1000

[18] Gaztañaga, E. (2021) Inside a Black Hole: the illusion of a Big Bang. https://hal.archives-ouvertes.fr/hal-03106344

[19] Gaztañaga, E. (2021) The Cosmological Constant as a Zero Action Boundary. Monthly Notices of the Royal Astronomical Society, 502, 436-444. https://doi.org/10.1093/mnras/stab056

[20] Le Corre, S. (2020) Negative Gravitational Mass: An Ideal Solution for Cosmology. Open Access Library Journal, 7, e6070. https://www.oalib.com/paper/pdf/5426416 